high workloads, rota gaps, and lack of a supportive environment.

Method Members of the Junior Doctors Representative Committee (JDRC) at University Hospitals Plymouth (UHP) NHS Trust, launched a bi-monthly initiative called Tea Break Talk. We put forward a proposal to our local BMA events organizer and arranged attendance of key people and services locally that we would like to promote as part of these sessions. Through open group discussions and questionnaires we assessed perceptions around seeking support from Clinical/Educational Supervisors and aim to support the wellbeing of junior doctors locally by signposting available services and peerled discussion/reflection.

Results Over a period of 3 months, we obtained feedback from doctors ranging from F1-ST6 levels. All responders found Tea Break sessions to be beneficial and a safe platform to discuss concerns regarding their wellbeing and professional development. $44 \%$ of the attendees were not aware of the existing counselling services, and only $48 \%$ felt comfortable discussing these stressful scenarios with their supervisors. Only one third of responders would discuss other personal or health-related concerns with their supervisors and almost $20 \%$ felt that doing so would reflect badly on them.

Conclusion Tea Break peer-led sessions have formed the ideal platform to facilitate reflection and promote wellbeing for Junior Doctors, as well as signposting information about alternative support systems available. Our data proves these sessions as a successful leadership initiative at Derriford Hospital and highlights the importance of establishment of similar initiatives in other hospitals in the South West.

\section{THE SLRP PROTOCOL: A TECHNIQUE FOR IMPROVING TELEPHONE TRIAGE FOR RECEPTIONISTS}

${ }^{1}$ Elizabeth Vincent, ${ }^{2}$ Imtiaz Ahmad. ${ }^{1}$ Kings College London, UK; ${ }^{2}$ South Lambeth Road Practice, UK

10.1136/leader-2019-FMLM.74

With the rising population number and age, and the current financial climate of the NHS, there is a greater burden on primary care services to create innovative solutions to meet the increasing patient demand. Alternative communication tools such as telephone triage have been proposed by the Royal College of General Practitioners (RCGP) as a costeffective, sustainable solution. The quality of the first patient interaction, typically conducted by receptionists, can have a substantial impact on patient care, yet most are not trained in medical communication. This project aimed to improve the telephone triage process at our practice (South Lambeth Road Practice) focusing specifically on the patient's first contact with receptionists as this is a potential area of significant risk. The involvement of a final year medical student in a leadership role was a novel aspect of this project that served as an invaluable link between medical and nonmedical staff.

A protocol entitled 'SLRP' (Symptoms, Length of symptoms, Recent actions, Priority) was created to aid reception staff with focused information gathering and effective triage.
Patient awareness was also increased by updating the practice website, phone service and production of posters.

Before telephone triage, $100 \%$ of calls resulted in face-toface consultations, compared to $45 \%$ after its implementation. Post-intervention there was a $142 \%$ rise in the quality of information gathered. Feedback from staff showed the protocol increased their confidence when interacting with and gathering information from patients.

This project has resulted in the implementation of further telephone triage days and interactive training sessions for receptionists. It highlighted the challenges faced by receptionists and has emphasised that effective leadership involves the ability to work collaboratively with non-medical and medical staff to create sustained improvement.

\section{WHAT IS THE ROLE OF A PHARMACIST IN A PARKINSON'S MULTIDISCIPLINARY TEAM?}

${ }^{1} \mathrm{~J} \mathrm{Dhap}{ }^{*},{ }^{2} \mathrm{~J}$ Morgan, ${ }^{2} \mathrm{~L}$ Breen, ${ }^{2} \mathrm{C}$ Powell. ${ }^{1}$ Worcestershire Health and Care NHS Trust, UK; ${ }^{2}$ University of Bradford

10.1136/leader-2019-FMLM.75

Worcestershire Integrated Neurological Rehabilitation Service (WINRS) recognised their Parkinson's disease (PD) caseload was increasing and more patients were presenting with complex care needs. A comparison of caseloads and referral times between healthcare professionals (HCPs) identified areas were workload was duplicated. Patients were also waiting longer for assessments with individual HCPs. WINRS collaborated with the Trusts Pharmacy Team to pilot PD multidisciplinary team (MDT) clinics.

Patients attend a PD MDT with the PD specialist nurse (PDSN), physiotherapist, occupational therapist (OT) and pharmacist in one single clinic to formulate a collaborative care plan. The PDSN leads the review using an agreed PD assessment tool ensuring a systematic review is conducted. Patients are given the opportunity to discuss their care needs and benefits from the expert knowledge of each HCP. The integrated care approach minimises duplication of workload and reduces waiting time referrals. The pharmacist supports the PDSN with accurate medication history, medication review and any other medication-related problems.

The utilisation of pharmacists' specialist knowledge in long term conditions (LTCs) is highly recommended in national guidelines. However, a systematic literature search was conducted in 2018 using healthcare databases. The literature search identified 17 PD MDT peer-reviewed studies. The MDTs identified varied both in the number and the type of HCPs. Physiotherapists and OTs $(n=16)$ were included most often and PDSN $(n=8)$ included sometimes. None of the studies identified involved a pharmacist. Pharmacists have shown benefits when working in MDTs for other LTCs; however, their role in PD MDT is not researched enough.

Research is underway to identify the role of a pharmacist in a PD MDT. We anticipate the research will show that patients, carers and HCPs will prefer a more inclusive PD MDT with a pharmacist resulting in improved patient care. 